\title{
Measurements of Noise in Josephson-Effect Mixers
}

Robert J. Schoelkopf, Jonas Zmuidzinas, Member, IEEE, Thomas G. Phillips, Henry G. LeDuc, and Jeffrey A. Stern

\begin{abstract}
We present new heterodyne receiver results obtained at $100 \mathrm{GHz}$ using resistively-shunted $\mathrm{Nb}$ and $\mathrm{NbN}$ tunnel junctions. In addition, we have carried out accurate measurements of the available noise power of these devices at the $L$-band $(1.5 \mathrm{GHz})$ IF frequency. Both the heterodyne and the output noise measurements show that the noise of these devices can be a factor of five or more higher than that predicted by the simple current-biased RSJ model. The noise approaches the appropriate thermal or thermal and shot noise limits for bias voltages where the nonlinearity is not strong (i.e., $V>I_{C} R_{N}$ ), but as expected from the RSJ model, can be significantly higher at the low voltages where the mixers are typically biased. The bias voltage dependence of the noise shows structure which is associated with resonances in the RF embedding circuit. Surprisingly, we find that changes in the high-frequency $(100 \mathrm{GHz})$ impedance presented to the junction can dramatically affect the magnitude and voltage dependence of the low-frequency $(1.5 \mathrm{GHz})$ noise. This emphasizes the necessity of very closely matching the junction to free space over a wide frequency range.
\end{abstract}

\section{INTRODUCTION}

W HILE JOSEPHSON-EFFECT mixers have been shown to have low conversion loss and low local oscillator power requirements as millimeter-wave mixers, their sensitivity is not as good as SIS mixers which utilize the quasiparticle nonlinearity of superconducting tunnel junctions. Josephson mixers are known to suffer from an "excess" noise, whose origin and magnitude has been clarified by recent simulations based on the resistively-shunted junction model (RSJ) [1]. Recent progress in fabrication of high- $T_{C}$ Josephson devices (mostly of SNS weak link types) has produced $I_{C} R_{N}$ products of up to $10 \mathrm{mV}$ [2], [3]. These devices may be suitable for producing heterodyne receivers well into the terahertz frequency range. However, in order to draw conclusions about the expected sensitivity and technological potential of Josephson-effect mixers in this frequency regime, a firm understanding of the noise processes and their scaling will be required.

We have been engaged in studies of the noise which is present in numerical simulations using the RSJ model, and

Manuscript received July 15, 1994; revised November 15, 1994. This work was supported by NASA under Grant NAGW-107 and BMDO. R. J. Schoelkopf was supported by a NASA Graduate Student Researcher Fellowship.

R. J. Schoelkopf, J. Zmuidzinas, and T. G. Phillips are with Downs Laboratory of Physics, California Institute of Technology, Pasadena, CA 91125 USA.

H. G. LeDuc and J. A. Stern are with the Center for Space Microelectronics Technology, Jet Propulsion Laboratory, California Institute of Technology, Pasadena, CA 91109 USA.

IEEE Log Number 9409126. in measurements of the heterodyne performance of wellcharacterized, lithographically-controlled Josephson devices using $\mathrm{Nb}$ and $\mathrm{NbN}$ shunted tunnel junctions. The heterodyne measurements suggest that the output noise of the devices can be even larger than the "excess" predicted by the RSJ model. We have therefore undertaken accurate measurements of the output noise of these shunted junctions at the receiver intermediate frequency (IF) of $1.5 \mathrm{GHz}$, including calibration of the contributions of the IF amplifier and corrections for the power coupling to the junction.

\section{JUNCTION FABRICATION}

The devices used in the experiments described here were made using a process for resistively-shunted tunnel junctions described previously [4]. A submicron, high current-density tunnel junction [5], [6] is fabricated along with a AuGe resistor in close proximity. Junctions based both on $\mathrm{Nb} / \mathrm{AlOx} / \mathrm{Nb}$ and $\mathrm{NbN} / \mathrm{MgO} / \mathrm{NbN}$ trilayers are used. The shunt resistor reduces the junction normal-state resistance, as well as the $I_{C} R_{N}$ product, by a factor of typically three to five. The shunted junctions have normal resistances (after shunting) of $30-40 \Omega$, critical currents of $10-20 \mu \mathrm{A}, I_{C} R_{N}$ products of about $0.5 \mathrm{mV}$, and are very stable with time and thermal cycling. The current-voltage characteristics are non-hysteretic and very RSJ-like, the critical current scales with junction area, and the critical current can be modulated with a magnetic field. The junctions have current rises at the superconducting gap of the material $(2.9 \mathrm{mV}$ for $\mathrm{Nb}$ and approximately 4.5 $\mathrm{mV}$ for $\mathrm{NbN}$ ), but this effect is small, since most of the current flows through the shunt resistor. Because the shunting reduces the $I_{C} R_{N}$ product and thus the critical frequency $\left(\omega_{c}=2 e I_{C} R_{N} / \hbar\right)$, these devices should be nearly optimal for heterodyne detection at $100 \mathrm{GHz}$, but may not be suitable for higher frequencies.

\section{Heterodyne Receiver Measurements}

We have performed heterodyne detection tests using the shunted $\mathrm{Nb}$ and $\mathrm{NbN}$ junctions fabricated using the technique described above. The devices are fabricated on $100-\mu \mathrm{m}$-thick quartz substrates and mounted in a full-height rectangularwaveguide mixer mount. The mixer mount incorporates two mechanically-adjustable, non-contacting backshorts, which allow a wide variation of the impedance presented to the junction. The waveguide has a transition to a corrugated scalar feedhorn, whose beam is then coupled outside of the dewar 


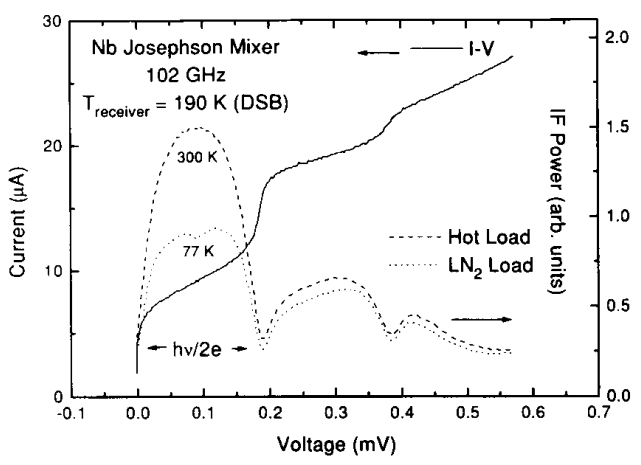

Fig. 1. Shows receiver response to hot/cold absorbers at input. Solid curve is $\mathrm{I}-\mathrm{V}$ curve under $102-\mathrm{GHz}$ LO radiation, dashed curve is IF output power (in arbitrary units) when receiver views a room temperature load, dotted curve is power when viewing an $l N_{2}$ soaked absorber. Horizontal axis is junction bias voltage in $\mathrm{mV}$. Only the positive half of the symmetric I-V curve is shown.

through a series of cooled IR filters and a polyethylene lens. Local oscillator (LO) radiation from a Gunn diode oscillator is injected into the beam through a mylar beam splitter. The beam pattern has been measured outside the dewar, and there is negligible spillover of the receiver beam onto dewar windows and filters. All measurements were performed with the devices cooled to $4.2 \mathrm{~K}$.

The junction is biased with a combination bias tee and intermediate frequency (IF) matching network, which provides an optimum match for impedances of about $90 \Omega$ into the $50-\Omega$ IF circuit. The IF signal is amplified by a HEMT lownoise amplifier (LNA) on a $12 \mathrm{~K}$ stage, which has a gain of $30 \mathrm{~dB}$ and noise temperature of approximately $10 \mathrm{~K}$. The amplifier bandpass is $500 \mathrm{MHz}$, centered at $1.5 \mathrm{GHz}$. The IF signal is then coupled to filters and room temperature amplifiers, and then finally led to a power detector. The bias electronics can be controlled using a personal computer, and current-voltage characteristics and IF power levels can be digitized and recorded for later analysis.

When illuminated with LO power, the devices develop strong Shapiro steps at the quantized voltages $V_{n}=n h \nu / 2 e$, and the IF power shows smooth peaks between the current steps (see Fig. 1). Receiver response is measured using the standard hot/cold load technique, typically with room temperature and liquid nitrogen cooled absorbers which fill the receiver beam outside the dewar. Best response is always obtained between the zeroth and first Shapiro steps, and for a local oscillator power which suppresses the critical current by approximately $50 \%$. All of this behavior is in agreement with the predictions of the RSJ model.

A representative pumped $\mathrm{I}-\mathrm{V}$ curve and hot/cold load response for a $\mathrm{Nb}$ shunted junction at $102 \mathrm{GHz}$ is pictured in Fig. 1. When biased at the optimum voltage, this receiver has a $\mathrm{Y}$-factor of 1.8 , and a double-sideband receiver noise temperature of $200 \mathrm{~K}$. The best sensitivity seen to date with $\mathrm{NbN}$ devices is $270 \mathrm{~K}$ (DSB), and the general receiver behavior is very similar for devices with either superconductor. The conversion efficiency is approximately $-6.5 \mathrm{~dB}$, and therefore the mixer noise temperature, including any front-end losses, was about $150 \mathrm{~K}$. Since this device was expected to be nearly optimal based on its observed dc characteristics, the mixer noise temperature expected from RSJ model simulations is closer to $30 \mathrm{~K}$ (DSB), with about $-3 \mathrm{~dB}$ conversion loss. Even when accounting for the slight saturation and possible single-sideband operation of the receiver as described below, and including estimates of the front-end optical losses, the mixer output noise is probably still a factor of 2-5 times higher than expected.

The mixer sensitivity is strongly dependent on the position of the adjustable RF tuners, with best response coming near the positions which yield the maximum output power, and also close to optimum LO power match into the device. If we measure the instantaneous bandwidth of the mixer by changing the LO frequency while keeping the tuner positions fixed, the noise temperature degrades by about $50 \%$ after the frequency has been displaced by the $3 \mathrm{GHz}$ separation between the sidebands, and then continues to increase roughly linearly with the frequency displacement. Note that this variation in receiver temperature is not due to compensation for the junction capacitance, as these devices have $\omega R C \approx 0.5$ at $100 \mathrm{GHz}$. This variation must instead be due to the intrinsic dependence of the mixer temperature on the RF port impedance. The observed dependence may indicate that the receiver is being tuned to a largely single-sideband mode, with a corresponding overestimate of the DSB mixer noise temperature from hot/cold load measurements.

We have also made a crude measurement of mixer linearity by plotting receiver output power as a function of input load temperature for liquid nitrogen, dry ice $(\cong 200 \mathrm{~K})$, room temperature, and heated $(\cong 350 \mathrm{~K}) \mathrm{RF}$ loads. The data indicate that the mixer is approximately $20 \%$ saturated for a room temperature input, and this effect probably accounts for a roughly $1 \mathrm{~dB}$ overestimate of the conversion loss. The size of this effect is reasonable given the experience with low-conversion-loss SIS mixers at these frequencies [7], [8]. Saturation probably arises in the output because the IF voltage swings are limited to the size of a photon step. One would expect that this effect should be significantly (up to a factor of 4) stronger in Josephson-effect mixers, since the photon steps are half as wide. The larger conversion loss, relative to SIS mixers, that we observe probably tends to reduce the saturation.

The shape of the pumped I-V curves can be varied radically as the mechanical tuners are adjusted. Several example curves for a single device under illumination with $98-\mathrm{GHz}$ LO radiation and varying tuner positions are shown in Fig. 2. The strong variation in output impedance verifies that a wide range of driving impedances can be presented to the junction. Negative differential resistance such as that seen in Fig. 2 is expected when the junction is driven with a largely inductive impedance [9], and the low output impedance probably corresponds to a capacitive drive. Both of these curves were taken for tuner positions which were far away from LO power match and therefore required significantly more LO power to yield equivalent suppression of the critical current. Best receiver performance is always observed for a more intermediate output impedance similar to that expected for a real drive impedance, which is again consistent with RSJ 


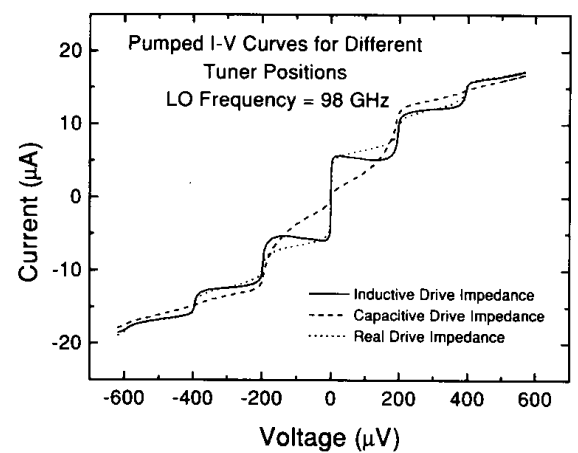

Fig. 2. Displays several $I-V$ curves of the same junction obtained under illumination with $98-\mathrm{GHz}$ local oscillator power. The different curves and the dramatically different dynamic resistances between photon steps are a result of the different positions used for the mechanical waveguide tuners. Differen amounts of LO power have been used to render approximately the same degree of critical current suppression. The full line probably corresponds to an inductive driving impedance, the dashed line to a capacitive drive, and the dotted line to a real drive impedance.

model predictions that optimum mixer performance should be possible with mostly real RF impedances.

\section{NoISE MEASUREMENT TEChNIQUE}

In order to verify that higher output noise is responsible for the degraded receiver sensitivity and to examine whether this noise can be reduced or eliminated, we undertook accurate measurements of available noise power produced by the junction under a variety of conditions, both with and without LO power applied. We report here only measurements in the absence of LO power, and with the optics removed so that the junction sees only $4.2 \mathrm{~K}$ thermal radiation at all frequencies. A block diagram of the microwave apparatus is displayed in Fig. 3. A coaxial switch [10] was inserted between the junction and the amplifier so that a variable temperature termination, similar to that described by McGrath et al. [11], could be substituted for the junction. Two isolators [12], each with a directivity of about $20 \mathrm{~dB}$, ensure that no reflected noise waves from the amplifier contaminate the measurements. Finally, a bi-directional coupler [13] allows a test signal to be introduced in order to measure the power reflection coefficient from the junction using a procedure described below. A similar technique has been used recently by Dubash et al. [14] to measure the output noise of SIS mixers to high accuracy.

By heating the termination and observing the output power as a function of temperature, the noise of the IF chain and the gain of the system could be determined. A typical calibration is displayed in Fig. 4. The termination was measured to have a return loss at cryogenic temperatures of about $-14 \mathrm{~dB}$. The chief uncertainty in calibration is in the accuracy of the diode temperature sensor [15] used to read the load temperature. The diode was calibrated by suspending it in a liquid helium storage dewar, and we estimate the error on the IF system noise determination to be $\pm 0.5 \mathrm{~K}$. The IF system temperatures were typically about $10 \mathrm{~K}$, varying slightly depending upon the bias conditions of the low noise amplifier and the bandpass selected by using room temperature filters.

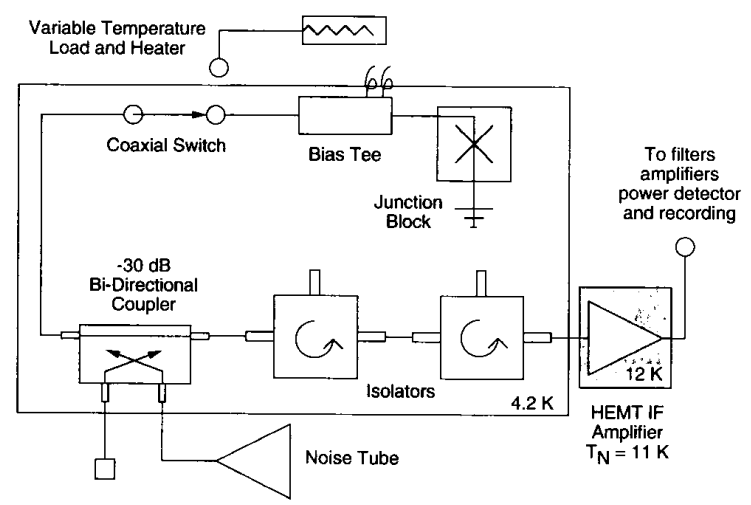

Fig. 3. A block diagram schematic of the apparatus used for noise measurerients. Junction is connected to a coaxial switch, through a bi-directional coupler and two isolators. Coupler allows calibration of junction reflection coefficient, and switch enables a variable-temperature termination to be connected to the IF input for calibration of system gain and noise.

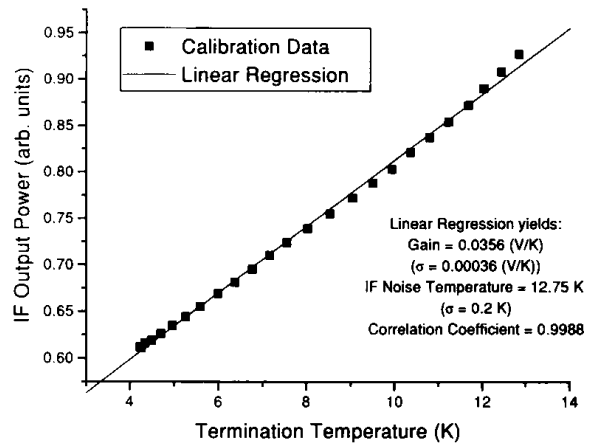

Fig. 4. A typical IF calibration using variable-temperature load. Horizontal axis shows measured load temperature, and output power in arbitrary units, corresponding to the voltage on total power detector, is displayed on vertical axis. Data points are shown along with best-fit linear regression to data and parameters corresponding to fit. Statistical errors for fit shown are determined by equally weighting all points and assuming that the deviations from linear fit represent the uncertainties. Fits are dominated by systematic errors (not shown).

To determine the available noise power from the junction and thus the junction output noise temperature, it is necessary to correct for any losses and mismatch between the junction and LNA. The bi-directional coupler allowed the introduction of a signal from a noise tube to be launched towards the junction. This signal was limited to a $500-\mathrm{MHz}$ band centered at the $1.5-\mathrm{GHz}$ IF frequency, and typically increased the total noise by about $20 \%$. No difference in the shape of the I-V curve was observed between noise tube on and off states, verifying that the noise did not grossly affect the junction properties. Because of the weak $(-30 \mathrm{~dB})$ coupling, very little noise is added when the noise tube is off, and merely causes an increase in the measured IF system temperature by a small $(<0.5 \mathrm{~K})$ amount. The directivity of the coupler at cryogenic temperatures was measured to be greater than $23 \mathrm{~dB}$.

The difference in IF power with and without the noise source applied gives a measure of the reflection coefficient. When the junction is biased in the zero-voltage state, where the dynamic resistance is zero, it can serve as a reference short-circuit. 
The power reflection coefficient of the junction, $\rho$, is therefore given by

$$
\rho(V)=\frac{P_{\mathrm{On}}(V)-P_{\mathrm{Off}}(V)}{P_{S \text {-On }}-P_{S \text {-Off }}}
$$

where $P_{\mathrm{On}}(V)$ is the power (as a function of the junction bias voltage, $V$ ) measured when the noise tube is on, and $P_{\mathrm{Off}}(V)$ is the measured power when it is off. The denominator, $P_{S \text {-On }}-P_{S \text {-Off }}$, is the difference between those powers when the junction is in the zero-voltage state; this quantity is proportional to the strength of the coupled test signal from the noise tube. By reversing the port of the coupler through which the test signal is introduced, we can measure any loss, $L$, between the coupler and junction

$$
L=1-\sqrt{\frac{P_{S-\mathrm{On}}-P_{S-\mathrm{Off}}}{P_{S-\mathrm{Rev}}-P_{S-\mathrm{Off}}}}
$$

where $P_{S-O n}$ and $P_{S-O f f}$ are as defined above, and $P_{S \text {-Rev }}$ is the power with the noise tube signal coupled in the reverse direction, towards the LNA. This loss was determined to be about $0.6 \mathrm{~dB}$, which is probably due to the small reflections in the coaxial switch and loss in the cabling to the bias tee. No correction for this loss is made to the estimate of the IF system temperature, which is measured including the switch. Any difference in the loss between the junction and the amplifier (for example, unequal cable lengths) relative to the loss between the variable load and the amplifier causes a small systematic error $(<0.4 \mathrm{~K})$ in the estimate of the amplifier contribution to the noise.

Having measured the IF reflection coefficient to the junction as a function of bias voltage, and having determined the IF system temperature through calibration with the variable temperature load, the available noise power or output noise temperature of the junction, in Kelvins, can be expressed as

$$
T_{\text {Junction }}=\frac{1}{(1-\rho(V))}\left(\frac{P_{\mathrm{Off}}(V)}{G}-T_{I F}-\rho(V) T_{\text {Physical }}\right)
$$

where $P_{\text {Off }}(V)$ is the measured IF system output with only the junction's noise at the input, $G$ and $T_{I F}$ are the measured IF system gain and noise temperature, and $T_{\mathrm{Physical}}$ is the physical temperature of the isolators, whose terminations radiate thermal noise towards the junction which is partially reflected back into the LNA. The errors in the junction noise temperature are dominated by systematic errors in the determination of the IF system temperature, and in systematic errors in the measurement of the reflection coefficient due to the finite directivity of the directional coupler, which are more severe for large reflection coefficients.

\section{JUNCTION NOISE MEASUREMENTS}

The apparatus and techniques described above have been used to measure the output noise temperature of both $\mathrm{Nb}$ and $\mathrm{NbN}$ shunted junctions. A plot of $T_{\text {junction }}$ as a function of bias voltage for a $\mathrm{NbN}$ device with a magnetic field applied is shown in Fig. 5. This curve displays several representative features seen in all of the measurements. First, the noise temperature above voltages comparable to the $I_{C} R_{N}$ product

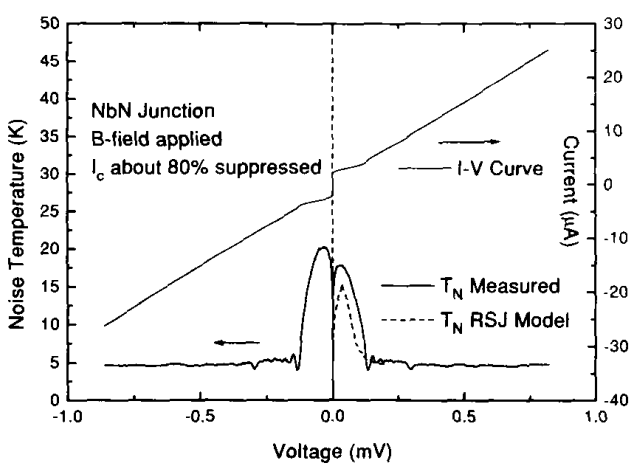

Fig. 5. Measured junction noise temperature and value calculated from RSJ model are displayed as a function of bias voltage. The parameters for the RSJ simulation have been derived from fitting the $\mathrm{dc} I-\mathrm{V}$ curve, and assuming only thermal noise at $4.2 \mathrm{~K}$ is input into model. An external magnetic field has been applied to suppress the critical current to about one-fifth its usual value. The dc supercurrent occurs at zero voltage, in the center of the horizontal axis. This data shows behavior similar to the RSJ model predictions, and approaches a value consistent with thermal noise at voltages above about $0.5 \mathrm{mV}$.

(in this case, about $100 \mu \mathrm{V}$ ), where the junction I-V curve is linear but below the superconducting gap of the underlying tunnel junction, is nearly constant with a value of about 4.6 $\mathrm{K}$. One would expect that in this region the noise would be mostly Johnson noise with a noise temperature equal to the physical temperature of $4.2 \mathrm{~K}$. Other devices show similarly increased noise temperatures in this region, which could be due to systematic errors in the measurements, a slightly elevated temperature for the device itself, or perhaps some shot noise due to subgap leakage of the tunnel junction. Above the gap voltage of the junction (not shown), the noise temperature increases linearly with bias voltage (or current), and the slope of this line is approximately $1.5-2 \mathrm{~K}$ per $\mathrm{mV}$ of bias voltage. This is as expected for our devices from shot noise. Recalling that the junction has been shunted with a normal resistor such that its resistance is reduced, the circuit at high voltages should be a normal resistor with Johnson noise (not bias-dependent) in parallel with a shot-noise-producing tunnel junction. Therefore the noise should rise linearly with voltage, but with a slope reduced from the full shot-noise value ${ }^{1}$ of $5.8 \mathrm{~K} / \mathrm{mV}$ by the same factor of three to five as the resistance has been reduced. Both the observed noise floor and the behavior above the gap confirm that the calibration and measurement procedure are accurate to within the expected systematic uncertainties.

There is also a large noise spike, either positive or negative, located exactly at zero bias voltage. In all cases, the noise at zero bias is consistent with the contribution of the IF system alone. However, the coupling of the junction also vanishes (by definition) at this point, where the dynamic resistance is zero, so that the available power at this point is undefined. This divergence can be ignored and is not of consequence for any of the discussions below. Finally, one notices a substantial

'The "effective shot noise temperature" is actually given by taking the current spectral density for pure shot noise, $S_{I}=2 \mathrm{eI}$. and comparing with the current spectral density for thermal noise, $S_{I}$ (Johnson) $=4 k b T / R$, giving a noise temperature, $T_{\text {shot }}=2 e I R / 4 k_{b}$. For a tunnel junction with no significant excess current in the linear region above the gap, however, $I$ times $R$ is just the bias voltage, so that $T_{\text {shot }}$ becomes $\mathrm{eV} / 2 k_{b}$. or $5.8 \mathrm{~K} / \mathrm{mV}$. 
asymmetry of approximately $10 \%$ in the maximum noise value attained by the junction at equivalent positive and negative bias voltages. This asymmetry is orders of magnitude larger than the statistical errors in the measurement, and is reproducible and not dependent on the direction in which the voltage is swept. However, the sign of the asymmetry (i.e., whether the higher peak is at positive or negative voltage) can easily be reversed by changing the direction of the externally applied magnetic field. This effect must therefore be a self-field effect due to the addition of the external field with the field caused by the current through the junction or shunt resistor. The current-voltage characteristics between the two states also show small related asymmetries.

The most important feature seen in the plots are the smooth maxima in output noise temperature seen at both positive and negative voltages. These peaks occur at voltages where the device is strongly nonlinear (i.e., $V$ less than about $I_{C} R_{N}$ ), and are due to noise which is converted downwards in frequency due to the Josephson effect, and also to power from the tails of the ac Josephson oscillations which can appear at the IF frequency. A theoretical curve which is obtained from the RSJ model with thermal noise, using the parameters determined from the best fit to the $\mathrm{I}-\mathrm{V}$ curve, is shown for comparison. It compares relatively well with the observed behavior, showing roughly the correct magnitude for the noise and reproducing the dependence on bias voltage. Some of the sharp ripples in the experimental data are not expected, and these are related to small ripples in the $\mathrm{I}-\mathrm{V}$ curve which are not present in the RSJ model.

Another similar set of curves, in which the magnetic field and the critical current suppression are minimal, are displayed in Fig. 6. Here the junction noise temperature attains much larger values before settling to the same thermal noise floor. In addition, the noise shows departures from the smooth, single maximum on either side of zero voltage which is always predicted by the RSJ model. The features in the noise again seem to be correlated with voltages where there are resonances and variations in the dynamic resistance of the junction, as seen in the current-voltage characteristic. Similar features are seen in the IF reflection coefficient of the junction, but the noise temperatures have been corrected for this variation. There must therefore be a variation in the output noise. In addition, the RSJ model predicts a maximum output noise of only about 10 times the physical temperature, or about $50 \mathrm{~K}$, for realistic parameter values corresponding to this junction. The observed noise is therefore seen to be a factor of five to ten times higher than expected, which is roughly consistent with the conclusions drawn from the heterodyne measurements of these devices. While the details of the shape of the curve depend on the junction parameters, this type of behavior, i.e., large noise and strong, non-RSJ variations with bias voltage, have been observed in several devices and both superconducting materials systems investigated.

The four superposed curves in Fig. 6 are taken with the same $\mathrm{NbN}$ junction on subsequent cooldowns. In between cooldowns, the choke structure for the waveguide system which is deposited along with the junction onto the quartz substrate was trimmed away. This choke structure consists

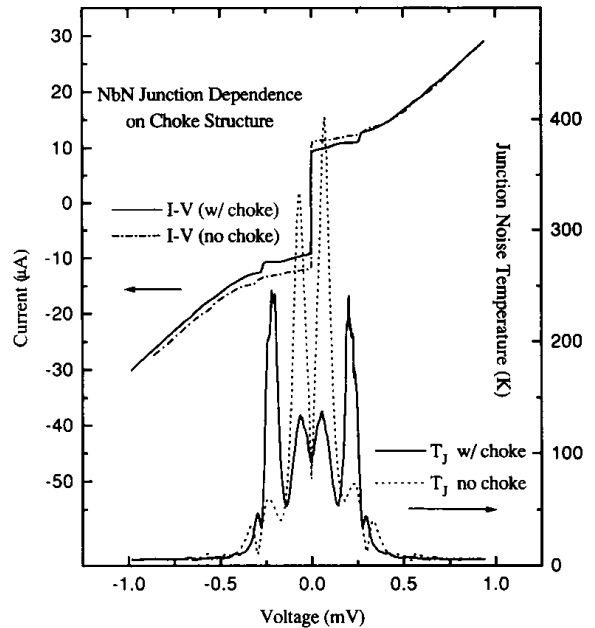

Fig. 6. Shows the effect of removing the RF choke from an NbN junction. Left axis is current, and the full and dash-dotted curves show the I-V curves on subsequent cooldowns; full line is with the choke, dash-dotted is without choke. A slight variation in the amount of external magnetic field is responsible for the discrepancy in critical current. The removal of the choke has weakened the resonance visible at about $0.3 \mathrm{mV}$, and displaced it slightly. Right axis displays the junction effective noise temperature (in $\mathrm{K}$ ); the spikes at zero voltage (see text) have been removed for clarity. Full line is with choke present, dotted line is after choke removal. Notice the change in the maximum value attained by the noise, and the qualitative change in the shape (i.e., largest and second-largest peak change location).

of an alternating series of microstrip sections which are approximately a quarter-wavelength at $100 \mathrm{GHz}$, and serve to couple the device to the waveguide. Most of the first section remained in order to provide a location to contact the junction. Comparison of the I-V curves (although there are slightly different amounts of trapped magnetic flux in the two cases) show that the strong resonance at about $0.4 \mathrm{mV}$ has been weakened and moved to slightly higher voltage. Surprisingly, the shape of the junction noise temperature has also been altered, and the distinctive double-humped shape could not be reproduced for any applied magnetic field. Again we see that the shape of the I-V curve, the presence of resonances, and the low-frequency output noise of the device can be strongly influenced by the RF impedance presented to the junction at fairly high frequencies. This dependence must be due to the nonlinear interaction of the junction and its embedding circuit. Of course the I-V curve will be dramatically different under LO illumination, and the changes in dynamic resistance due to the appearance of the Shapiro steps will alter the dependence of the noise on voltage, tending to smear out some of the resonances. The noise of the junctions when weakly coupled to an RF source has been measured, and will be reported elsewhere.

\section{DISCUSSION AND CONCLUSION}

We have performed heterodyne measurements using shunted tunnel junctions at $100 \mathrm{GHz}$, which show fairly good performance, but suggest that several non-ideal effects, including weak saturation and possible single-sideband operation, are present. There is also a strong dependence of receiver 
performance and dynamic resistance on the RF embedding impedance. Most importantly, however, these heterodyne measurements indicate that the receiver performance is probably degraded because of elevated output noise from the devices. While the RSJ model predicts that the junction noise should be a factor of five to ten above the thermal noise limit (i.e., $T_{N}$ for the junction is several times $\left.T_{\text {Physical }}\right)$, we observe noise levels under some conditions which are higher than the RSJ predictions by another factor of about five.

Accurate measurements of the junction output noise at the intermediate frequency of $1.5 \mathrm{GHz}$ have been performed to verify this hypothesis. The output noise of the junctions, even without local oscillator power applied, are observed to attain values higher than predicted from the RSJ model, and also show a complicated behavior with bias voltage which cannot be reproduced without inclusion of the external circuit into the nonlinear modeling of the RSJ circuit. We have shown that the low-frequency $(1 \mathrm{GHz})$ output noise, and its voltage dependence, can be radically altered simply by changing the high frequency $(100 \mathrm{GHz})$ impedance presented to the junction. Inspection of the I-V curves shows that the peaks in junction noise temperature are correlated with regions of high dynamic resistance, where the Josephson oscillation linewidth, and hence the "excess" noise, according to our picture for its generation [1], might be expected to be at a maximum.

It remains somewhat unclear what the implications are for the development of low-noise Josephson-effect mixers. Elevated output noise, if only a factor of five to ten times the physical temperature, would not preclude interestingly low mixer temperatures, particularly at the higher frequencies where SIS mixers are not yet available. However, our measurements show that the noise can be sensitive to the RF design of the mixer and to unanticipated resonances at high frequencies. Antenna-coupled mixer designs which are able to present a well-behaved, real impedance over a wide frequency range (e.g., self-similar structures such as log-periodic spirals [16]) should probably be used in the future.

\section{ACKNOWLEDGMENT}

The authors would like to thank N. Dubash, R. McGrath, and T. Kerr for helping to find suppliers of microwave equipment suitable for low temperatures, J. Kooi and P. Schaffer for assistance with design and fabrication of the cryostat, and G. Ugras, E. Grossman, and T. Groesbeck for helpful discussions.

\section{REFERENCES}

[1] R. J. Schoelkopf, J. Zmuidzinas, and T. G. Phillips, in preparation; R. J Schoelkopf, Ph.D. dissertation, California Institute of Technology, 1995.

[2] P. A. Rosenthal, E. N. Grossman, R. H. Ono, and L. R. Vale, Appl. Phys. Lett., vol. 63, pp. 1984-1986, 1993.

[3] P. A. Rosenthal and E. N. Grossman, IEEE Trans. Microwave Theory Tech., in press.

4] R. J. Schoelkopf, T. G. Phillips, and J. Zmuidzinas, IEEE Trans. Appl. Supercond. vol. 3 , pp. 2250-2253, 1993

[5] H. G. LeDuc et al., "Submicron area Nb/A1Ox/Nb tunnel junctions," in Proc. 3rd Int. Symp. Space Terahertz Technol., Ann Arbor, MI, Mar. 1992

[6] J. A. Stern, H. G. LeDuc, and A. J. Judas, "Fabrication and characterization of high current-density, submicron $\mathrm{NbN} / \mathrm{MgO} / \mathrm{NbN}$ tunne junctions," in Proc. 3rd Int. Symp. Space Terahertz Technol., Ann Arbor, MI, Mar. 1992
[7] L. D'Addario, Intl. J. IR and MM Waves, vol. 5, pp. 1419-1442, 1984.

[8] D. Woody, private communication.

[9] Y. Taur, IEEE Trans. Electron Dev., vol. ED-27, pp. 1921-1928, 1980.

[10] SPDT latching coaxial switch, part \#909C70200, Daytron/Transco, Simi Valley, CA

[11] W. R McGrath, A. V Räisänen, and P. L. Richards, Int. J. IR and MM Waves, vol. 7, pp. 543-553, 1986

[12] Pamtech 1.25-1.75-GHz isolator, Pamtech, Inc., Canoga Park, CA

[13] Directional coupler, model DCS-305, Technical Research and Manufacturing, Inc., Bedford, NH.

[14] N. B. Dubash, G. Pance, and M. J. Wengler, "Photon noise in the SIS detector," in Proc. 4th Int. Symp. Space Terahertz Technol., Los Angeles, CA, Apr. 1993.

[15] Diode temperature sensor model DT-500, Lake Shore Cryotronics Inc., Westerville, $\mathrm{OH}$.

[16] T. H. Buettgenbach et al., IEEE Trans. Microwave Theory Tech., vol MTT-36, pp. $1720-1726,1988$.

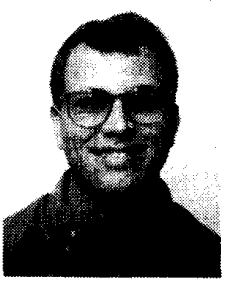

Robert J. Schoelkopf (M'53-SM'79-L S'86) received the A.B. degree in physics (cum laude) from Princeton University in 1986 . His undergraduate thesis involved a search for acceleration-dependent quantum phase effects in superconductors.

He spent two years at the Laboratory for HighEnergy Astrophysics, NASA/Goddard Space Fligh Center, where he worked on the development of cryogenic microcalorimeters for X-ray spectroscopy and cryogenic design for spacecraft. In 1988, he entered the graduate program in the physics department at the California Institute of Technology, where he has worked on superconducting mixers for submillimeter-wave detection. His research interests include galactic X-ray binaries, nonlinear dynamics, and novel lowtemperature detectors.

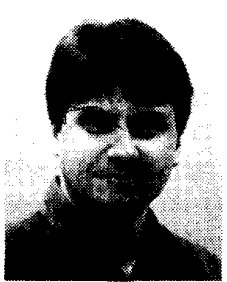

Jonas Zmuidzinas (M'91) received the B.S. degree in physics from the California Institute of Technology in 1981, and the Ph.D. degree in physics from the University of California, Berkeley, in 1978. His $\mathrm{Ph} . \mathrm{D}$, thesis described the development of a laser heterodyne receiver for airborne astronomy in the far-infrared $(800-2000 \mathrm{GHz})$

During 1988-1989 he was a postdoctoral fellow at the University of Illinois, where he worked on the design and fabrication of SIS mixers. He is currently an assistant professor of physics at the California Institute of Technology. His research interests are in the areas of high-frequency superconducting devices and their application to submillimeter astronomy.

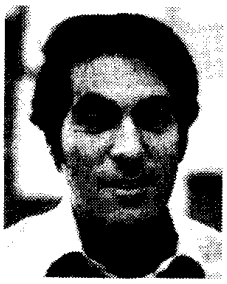

Thomas G. Phillips was educated at Oxford, England, where he received B.A., M.A., and D.Phil. degrees. His graduate studies were in low-temperature physics.

After one year at Stanford University, he returned to Oxford for two years and then moved to the Bell Laboratories Physics Research Laboratory at Murray Hill, NJ. There he developed techniques for millimeter and submillimeter-wave detection for astronomy. In 1975 he spent one year at London University as university reader in physics. In 1980 he joined the faculty of Caltech as professor of physics. At Caltech he took on the task of construction of the Owens Valley Radio Observatory millimeterwave interferometer, as Associate Director of the Observatory. In 1982 he became Director Designate for the Caltech Submillimeter-wave Observatory, to be constructed in Hawaii, and in 1986, on successful completion of the construction, became Director. His current research interests are in molecular and atomic spectroscopy of the interstellar medium and in the development of superconducting devices for submillimeter-wave detection. 
Henry G. LeDuc received the B.S. degree in physics from Montana State University, Bozeman, MT, in 1977, and the Ph.D. degree in physics from the University of California, Davis, in 1983. His thesis work involved far-infrared Jeffrey A. Stern, photograph and biography not available at time of publispectroscopy of solid state ionic conductors.

At present, he is a group leader at California Institute of Technology's Jet Propulsion Laboratory. His group is developing SIS tunnel junctions for heterodyne receivers. 